\title{
Genetic variance and covariance patterns for body weight and energy balance characters in an advanced intercross population of mice
}

\author{
Larry J. LEAMY ${ }^{\mathrm{a} *}$, Kari ELO ${ }^{\mathrm{b} * *}$, Merlyn K. NIELSEN $^{\mathrm{b}}$, \\ L. Dale VAN VLECK ${ }^{\mathrm{c}}$, Daniel POMP ${ }^{\mathrm{b}}$ \\ a Department of Biology, University of North Carolina at Charlotte, Charlotte, \\ NC 28223, USA \\ ${ }^{\mathrm{b}}$ Department of Animal Science, University of Nebraska, Lincoln, NE 68583, USA \\ ${ }^{\mathrm{c}}$ Roman L. Hruska U.S. Meat Animal Research Center, ARS, USDA, Lincoln, \\ NE 68583, USA
}

(Received 12 May 2004; accepted 9 July 2004)

\begin{abstract}
We estimated heritabilities and genetic correlations for a suite of 15 characters in five functional groups in an advanced intercross population of over 2000 mice derived from a cross of inbred lines selected for high and low heat loss. Heritabilities averaged 0.56 for three body weights, 0.23 for two energy balance characters, 0.48 for three bone characters, 0.35 for four measures of adiposity, and 0.27 for three organ weights, all of which were generally consistent in magnitude with estimates derived in previous studies. Genetic correlations varied from -0.65 to +0.98 , and were higher within these functional groups than between groups. These correlations generally conformed to a priori expectations, being positive in sign for energy expenditure and consumption $(+0.24)$ and negative in sign for energy expenditure and adiposity $(-0.17)$. The genetic correlations of adiposity with body weight at 3,6 , and 12 weeks of age $(-0.29,-0.22,-0.26)$ all were negative in sign but not statistically significant. The independence of body weight and adiposity suggests that this advanced intercross population is ideal for a comprehensive discovery of genes controlling regulation of mammalian adiposity that are distinct from those for body weight.
\end{abstract}

advanced intercross mice / body weight / genetic correlations / heritability / obesity

\footnotetext{
*Corresponding author: 1jleamy@email.uncc.edu

** Present address: Department of Animal Science, University of Helsinki, 00014 Helsinki, Finland
} 


\section{INTRODUCTION}

Body size, weights of various organs, adiposity, bone mineral density, heat production, feed intake, and many other complex characters in mammals all have a polygenic mode of inheritance and thus are expected to be governed by the action and interaction of many genes [23,30]. This has been amply demonstrated in recent years with the identification of increasing numbers of quantitative trait loci (QTL) affecting these characters $[5,6,11,13,15,26,35$, 37,43,44]. Most of these QTL studies have been conducted with mice because of the availability of inbred strains or selected lines that are chosen or bred for divergence in the mean of the character of interest and crossed to yield individuals in the $F_{2}$ generation with genotypic variability at a number of loci controlling the character [14].

The QTL found in these studies often show pleiotropic effects [13,35], especially for characters that are functionally or developmentally related $[8,12,34]$. Pleiotropy is largely responsible for generating genetic correlations between characters [23], with the magnitude of these correlations often reflecting the degree of pleiotropy. A relatively high genetic correlation $(+0.60)$ between body weight and adiposity has been estimated in mice [20], for example, and several studies have found that a number of QTL for body weight apparently also affect adiposity $[13,44]$. Body weight itself actually is a composite character that is expected to be correlated with many of its parts (organ weights, etc.), and some studies have adjusted for weight to search for QTL affecting the characters that are independent of overall size $[37,48,49]$. In such analyses with adjusted characters, we would expect fewer instances of pleiotropy and generally lower levels (and perhaps different patterns) of genetic correlations than in analyses using characters not adjusted for body weight.

Sorting out patterns of genetic correlations involving a number of body composition and energy balance characters is critical to our understanding of the role genes play in the formation of complex characters [39]. Adiposity, for example, often is associated with weight gain due to an energy imbalance in which energy intake exceeds energy expenditure [50], and genetic correlations of adiposity with various energy measures would be useful to test the magnitude of these associations. We also need estimates of the genetic association of adiposity with weight at different ages since different subsets of QTL apparently influence early and late growth in mice $[11,44,48]$. Adiposity does not appear to be highly related to the skeletal system in mice, at least as measured by tail length $[13,29]$. But we know little of the genetic linkage of adiposity with either bone mineral content or bone mineral density, although these bone characters are associated with weight change in humans [46]. 
The purpose of the study described in this paper was to calculate genetic parameters (heritabilities and genetic correlations) for a total of 15 characters in five (adiposity, body weight, energy, bone, and organ weight) groups in order to understand their patterns of genetic variability and covariability. All characters were measured in over 2000 mice from an advanced intercross population derived from a cross of lines originally selected for low (ML) and high (MH) heat loss $[41,42]$. This population was developed for an eventual fine mapping analysis of QTL, and it was useful to first estimate heritabilities and genetic correlations to serve as a basis for comparison with the QTL results.

In addition, we estimated these genetic parameters for the 15 characters in order to test several expectations. Specifically, we wished to test whether genetic correlations would be higher within than between the five groups, as expected if the characters in each of these groups are functionally integrated $[8,12]$. We also were interested to know whether our overall measure of adiposity (total body fat percentage) would be highly genetically correlated with three other measures of adiposity often used to assess overall fatness [44]. In addition, because our mice were derived from selection lines whose heat loss and feed intake measures exhibited opposite patterns to that of body fat $[36,42]$, we predicted that the genetic correlation of heat loss would be positive with feed intake but negative with the adiposity measures. We tested these and other expectations, and compared phenotypic and genetic correlations for all characters, adjusting and not adjusting for body weight in 10 of these characters.

\section{MATERIALS AND METHODS}

\subsection{Resource population}

The resource population (AIL-F11) of mice used in this study was originally derived from lines selected for low (ML) and high (MH) heat loss [44]. After 16 generations of selection, these lines achieved a divergence of $\sim 50 \%$ in heat loss, or direct responses (expressed as deviations from the unselected control line mean of $134.9 \mathrm{kcal} / \mathrm{kg}^{0.75} /$ day) of $44.2 \mathrm{kcal} / \mathrm{kg}^{0.75} /$ day for $\mathrm{MH}$ and $-27.4 \mathrm{kcal} / \mathrm{kg}^{0.75} /$ day for ML [44]. Further, the divergence between MH and ML was $20.6 \%$ for feed intake per unit metabolic size, and $40 \%$ for body fat percentage, with $\mathrm{MH}$ mice consuming more energy while possessing less body fat than ML mice [36]. Selection in these lines also significantly affected litter size and ovulation rate [42] as well as locomotor activity and core body temperature [38]. Specifically, $\mathrm{MH}$ mice were twice as active, and had a core temperature that averaged $0.51{ }^{\circ} \mathrm{C}$ higher, than ML mice. 
We first sampled mice from the $\mathrm{MH}$ and ML lines and inbred them via full-sib mating for seven generations to establish inbred high (MHI) and low heat loss (MLI) lines. An intercross of the MHI and MLI lines was then made and continued for 11 generations to produce an advanced intercross line, or AIL [17]. During each intercrossing generation, we used a cyclical mating scheme in an effort to minimize inbreeding. Breeders were drawn at random from 30 litters (30 males and 60 females) each generation, with individual males crossed with two females that were sisters. One litter from each pair of sisters was chosen to be reared for the next generation of breeders. At intercross generation 8, the AIL was divided into two replicates and at generation 10 into four replicates. In generation 10, each mating was replicated, producing a total of 2075 mice in 8 different groups (4 replicates each with 2 parities) in generation 11. The production of AIL-F11 is schematically presented in Figure 1. Pedigree information was recorded starting from the parental mating (generation $0, \mathrm{G} 0$ ) but in the present analysis we used information only from generations 7 to 11 since the relative contribution of earlier generations to the overall amount of inbreeding achieved was considered marginal.

In all generations of these crosses, mice were housed in plastic cages (limit of 4 mice per cage) with wood-chip bedding, had ad libitum access to water and feed (Teklad 8604), and were maintained at a temperature of $22{ }^{\circ} \mathrm{C}$ (relative humidity of $35-50 \%$ ) and at a light:dark cycle of $12: 12$ hours. In the generation 11 intercross (AIL-F11), all litter sizes were standardized to 8 pups at birth and pups were weaned at 3 weeks of age and weighed (WT3W) in grams on a digital scale (Mettler, Columbus, $\mathrm{OH}$ ). Additional body weights were taken for each mouse at 6 (WT6W) and 12 (WT12W) weeks of age.

\subsection{Measurement protocols}

Heat loss (HL) was measured in the AIL-F11 mice at an average age of 13 weeks in direct, gradient-layer, individual-animal calorimeters (Thermonetics Corporation, San Diego, CA) as previously described [41]. Heat energy was assessed as a change in the electric voltage passing through the walls and sensors of the calorimeters. Average heat loss over a $15 \mathrm{~h}$ period was adjusted for metabolic weight and time ( $\mathrm{kcal} / \mathrm{kg}^{0.75} /$ day). While in the calorimeter, each mouse was given a $3.0 \mathrm{~g}$ feed pellet and the amount of feed left at the end of the measurement period was recorded.

Feed intake (FI) was measured for a period of seven days starting after calorimetric measurement. Each mouse was individually caged and at the beginning of the trial received an amount of feed equal to about two times the 


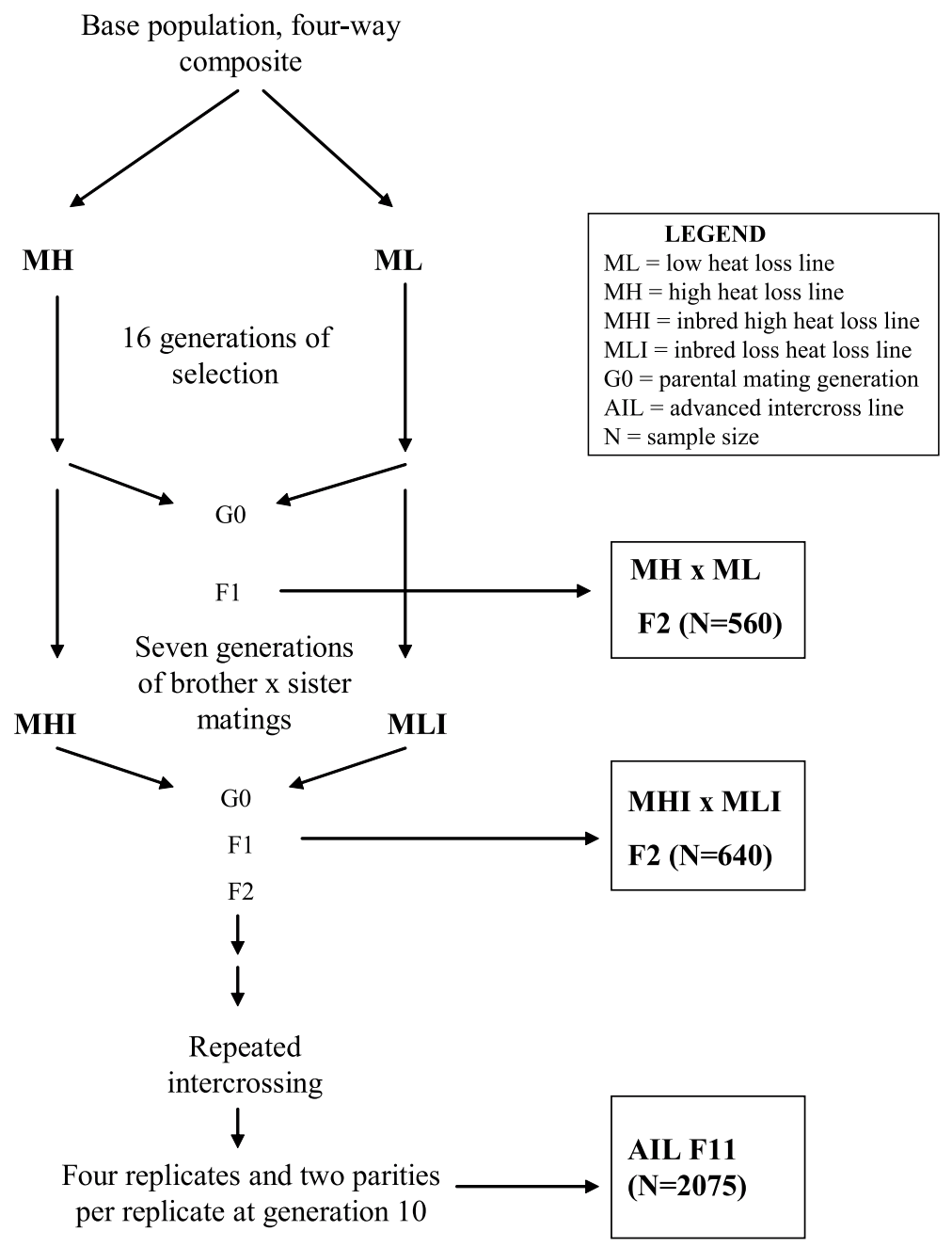

Figure 1. Production of the advanced intercross (AIL-F11) population of mice used in this study.

average weekly consumption ( $~ 90$ grams). Feed weights were recorded in the beginning and the end of the feed intake trial and were used to calculate feed intake in grams calculated per day, per day per average body weight during the feed intake trial, and per day per average metabolic body weight. Because all three measures gave similar results in a preliminary analysis, we used feed intake expressed per average metabolic body weight $\left(\mathrm{g} / \mathrm{kg}^{0.75} /\right.$ day) to be consistent with the units used for HL. 
All mice were weighed and euthanized at an average age of 15 weeks and the entire body except the head (the subcranial region) was scanned in a consistent, dorsal position [40] using a dual-energy X-ray absorption (DEXA) densitometer (PIXImus, Lunar Corporation, Madison, WI). The DEXA measurements estimated four body composition characters in each mouse: total subcranial fat (FAT) in $\mathrm{g}$, bone mineral density (BMD) in $\mathrm{g} / \mathrm{cm}^{2}$, bone mineral content (BMC) in $\mathrm{g}$, and bone area (BA) in $\mathrm{cm}^{2}$. We also recorded the $\mathrm{R}$ values from the PIXImus scans since this is a standard measure of "non-fat" or "lean" tissue, but this measure was nearly perfectly negatively correlated with FAT and was therefore eliminated as redundant. After each mouse was scanned, we dissected and weighed (all in grams) its brown adipose tissue (BAT), right hindlimb subcutaneous fat pad (SCF), right gonadal (male) or perimetrial (female) fat pad (GF), spleen (SPLEEN), liver (LIVER) and heart (HEART).

Altogether, 15 characters were analyzed: 3 body weights, heat loss, feed intake, the 4 DEXA phenotypes, and 6 fat pad and organ weights. There were some original technical problems with the PIXImus that were eventually solved, but this slightly reduced the sample size for several of the characters from the original total of 2075. In addition, occasional mice were missing or died prematurely, some characters could not be measured, and a few recording errors were discovered (some were correctable) after screening. As a consequence, the total sample sizes for each character varied from 1935 for the three bone characters (BMD, BMC, BA) to a maximum of 2040 (see Tab. I).

\subsection{Statistical analysis}

The data for all 15 characters were fitted to an animal model with multipletrait derivative-free restricted maximum-likelihood (MTDFREML) procedures $[4,24]$ in order to estimate genetic and environmental components of variance/covariance. Several fixed classification effects (including sex) and covariates were included in this model. Preliminary analyses were run for the separate sexes, but genetic parameter estimates for males and females showed high congruence and thus we combined both sexes in this analysis. Besides sex, groups (4 replicates $\times 2$ parities), the inbreeding coefficient of the individual, and the inbreeding coefficient of the female parent (dam) were used as classification effects for all 15 characters. The inbreeding coefficients were calculated by MTDFREML as continuous variables for individuals and dams that assumed several values from 0 to 0.219 , making it convenient to convert them to discontinuous dummy variables for use rather than treat them as continuous covariates in the model. For HL, two additional classification effects 
were used for each mouse: its age when the heat loss measurement started, and the specific calorimeter unit number it was placed in for this measurement. In addition for HL, the amount of feed left after the calorimeter measurement was used as a linear covariate. For the 10 characters measured from DEXA (BMD, BMC, BA, FAT) and dissections (BAT, SCF, GF, SPLEEN, LIVER, HEART), the age at sacrifice and the day of dissection were included as classification effects. These 10 characters also were analyzed both with and without body weight at sacrifice as a covariate (linear or quadratic as appropriate) to determine the effects of adjustment for the overall weight of the mice on the variance/covariance estimates. Throughout the analysis and discussion below, adjusted characters are indicated with an 'a' superscript (e.g. BAT').

Beyond these fixed classification and covariate effects, the basic animal model included additive (direct) genetic effects, and two uncorrelated random effects: maternal (dam) effects and litter effects. The dam effects assessed both maternal genetic and maternal environmental sources of variance and covariance that affected all pups from both replicate litters produced by each dam. The litter variable identified each individual litter produced by a dam, and assessed any random environmental sources of variation/covariation among pups in a specific litter that were not accounted for by effects due to dams. We tested for significance of dam effects by calculating the difference between twice the log likelihood values obtained in MTDFREML runs conducted with and without this effect, and comparing this difference with the critical value for chi-square associated with 1 degree of freedom (3.84). Where effects due to the dam were not significant, we reran analyses without these effects. In implementing this model with the MTDFREML program, multiple starts were used to ensure that the algorithm was achieving a global rather than a local maximum.

For each of the 15 characters, MTDFREML estimated additive genetic, litter, dam (if significant), and residual environmental variances that also were expressed as a proportion of the total phenotypic variance. Although narrowsense heritabilities (additive genetic variance/total phenotypic variance) of each character were of greater interest than environmental components of variance, it was also useful to discover how much of the remaining environmental variance was common/not common to litters. Standard errors for all components were estimated by the delta method [19].

Estimation of phenotypic and genetic correlations also was done in MTDFREML for each of the 105 pairwise combinations of the 15 characters. The MTDFREML program was especially suitable for this since it can handle two characters that have different combinations of classification effects, covariates, 
and/or random effects. To estimate standard errors of the genetic correlation estimates, however, the program required that the samples be identical for both characters. We therefore used only those mice for which all 15 characters, classification effects, and covariates were present (sample size $=1766$ ). Once all correlations and their standard errors were calculated, we calculated $t$ statistics and their probabilities which then were evaluated with the sequential Bonferroni procedure $[25,47]$ to determine statistical significance.

The phenotypic and genetic correlation matrices were compared with Mantel's permutation test [18] in which one of these matrices was randomly permuted a number of times and a test statistic (Kendall's tau coefficient) calculated for each permutation. The null hypothesis of no similarity was then rejected if the number of test statistics with values equal to or greater than that calculated from the original matrices was less than $5 \%$ of all values obtained. This permutation test also was used to test for genetic integration within the five functional groups of characters by constructing a theoretical connectivity matrix consisting of $1 \mathrm{~s}$ for all within-group correlations and 0 s for betweengroup correlations and permuting this with the genetic correlation matrix. Rejection of the null hypothesis in this case would suggest that the within- and between-group correlations differed and that there is significant morphological integration $[7,10]$.

\section{RESULTS}

\subsection{Basic statistics and variance components}

Table I gives the sample size, mean, standard deviation, and coefficient of variation for each of the 15 characters after adjustment for all classification effects and covariates (including body weight at sacrifice) appropriate for each of these characters. The mean heat loss of $140.4 \mathrm{kcal} / \mathrm{kg}^{0.75} /$ day is slightly higher than the 134.9 value estimated by Nielsen et al. [44] for mice in the control lines in their selection experiment. The larger standard deviations are generally associated with characters with larger means, although coefficients of variation suggest that the bone characters $\left(\mathrm{BMD}^{\mathrm{a}}, \mathrm{BMC}^{\mathrm{a}}, \mathrm{BA}^{\mathrm{a}}\right)$ have the lowest amount of variability whereas $\mathrm{BAT}^{\mathrm{a}}, \mathrm{SCF}^{\mathrm{a}}, \mathrm{GF}^{\mathrm{a}}$, and SPLEEN ${ }^{\mathrm{a}}$ have the highest variability. WT3W, WT6W, WT9W, and HL also were measured in all mice in the $\mathrm{F}_{2}$ generation produced from crossing the MHI and MLI lines, and the means for these characters (Tab. I) are comparable to those in the AIL population.

Table II shows the genetic and environmental component estimates for the 15 characters, including estimates made with and without adjustment for 
Table I. Basic statistics for the 15 characters. Sample size $(N)$, mean, standard deviation (STD), and coefficient of variation (CV) for each of the 15 characters (with their units in parentheses and their abbreviations) used in the analysis. Standard deviations reflect variability in each character after adjustment for all appropriate fixed effects and covariates (see text). ${ }^{a}=$ adjusted for body weight at sacrifice. Means for four characters from the $\mathrm{F}_{2}$ population generated from crossing the MHI and MLI lines are given in parentheses.

\begin{tabular}{|c|c|c|c|c|c|}
\hline Character (units of measure) & Abbreviation & $N$ & Mean & STD & $\mathrm{CV}$ \\
\hline 3-week body weight (g) & WT3W & 2027 & $14.4(16.5)$ & 1.8 & 12.8 \\
\hline 6-week body weight (g) & WT6W & 2035 & $28.6(29.2)$ & 2.4 & 8.5 \\
\hline 12-week body weight (g) & WT12W & 2040 & $33.5(32.8)$ & 2.9 & 8.7 \\
\hline Heat loss ( $\mathrm{kcal} / \mathrm{kg}^{0.75} /$ day) & HL & 1973 & $140.4(135.7)$ & 16.1 & 11.4 \\
\hline Feed intake $\left(\mathrm{g} / \mathrm{kg}^{0.75} /\right.$ day $)$ & FI & 2001 & 83.9 & 14.1 & 17.8 \\
\hline Bone mineral density $\left(\mathrm{g} / \mathrm{cm}^{2}\right)$ & $\mathrm{BMD}^{\mathrm{a}}$ & 1935 & 0.06 & 0.003 & 5.6 \\
\hline Bone mineral content $(\mathrm{g})$ & $\mathrm{BMC}^{\mathrm{a}}$ & 1935 & 0.73 & 0.048 & 6.5 \\
\hline Bone area $\left(\mathrm{cm}^{2}\right)$ & $\mathrm{BA}^{\mathrm{a}}$ & 1935 & 11.77 & 0.57 & 4.9 \\
\hline Total body fat (g) & FAT $^{\mathrm{a}}$ & 2038 & 4.37 & 0.59 & 13.4 \\
\hline Brown adipose tissue $(\mathrm{g})$ & $\mathrm{BAT}^{\mathrm{a}}$ & 2033 & 0.05 & 0.010 & 22.9 \\
\hline Subcutaneous fat pad (g) & $\mathrm{SCF}^{\mathrm{a}}$ & 2040 & 0.13 & 0.032 & 25.5 \\
\hline Gonadal fat pad $(\mathrm{g})$ & $\mathrm{GF}^{\mathrm{a}}$ & 2040 & 0.16 & 0.064 & 40.3 \\
\hline Spleen weight (g) & SPLEEN $^{\mathrm{a}}$ & 2034 & 0.11 & 0.025 & 22.1 \\
\hline Liver weight (g) & LIVER $^{\mathrm{a}}$ & 2036 & 1.69 & 0.14 & 8.2 \\
\hline Heart weight (g) & HEART $^{\mathrm{a}}$ & 2035 & 0.18 & 0.024 & 12.9 \\
\hline
\end{tabular}

body weight for the 10 DEXA and dissection characters. Heritabilities average 0.56 for the three body weights, 0.23 for the two energy characters, 0.48 for the three bone characters ${ }^{\mathrm{a}}, 0.35$ for the four measures of adiposity ${ }^{\mathrm{a}}$, and 0.27 for the three organ weights ${ }^{\mathrm{a}}$. All estimates are significantly different from zero after appropriate adjustment for multiple comparisons with the sequential Bonferroni procedure [47]. Estimates tend to be highest in magnitude for the three body weight characters and two of the bone characters $\left(\mathrm{BMD}^{\mathrm{a}}\right.$ and $\mathrm{BMC}^{\mathrm{a}}$, but not $\left.\mathrm{BA}^{\mathrm{a}}\right)$, but low to moderate in magnitude for the remaining characters. Heritabilities estimated without adjustment for body weight all are higher $($ mean $=0.45)$ than those calculated with this adjustment (mean $=0.39)$, this increase being particularly noticeable for BMD, BMC, SPLEEN, LIVER, and HEART. 
Table II. Estimates of heritabilities, litter effects, maternal effects (where significant), and residual environmental effects \pm their standard errors for all 15 characters $\left({ }^{\mathrm{a}}=\right.$ adjusted for body weight at sacrifice) expressed as a proportion of the total phenotypic variance. Values in parentheses are estimates (standard errors not shown) of these same effects in characters not adjusted for body weight at the time of sacrifice.

\begin{tabular}{lllll}
\hline Character & Heritability & Litter Effect & Maternal Effect & Residual Environ. \\
\hline WT3W & $0.57 \pm 0.156$ & $0.27 \pm 0.048$ & $0.16 \pm 0.078$ & $0.00 \pm 0.085$ \\
WT6W & $0.53 \pm 0.137$ & $0.06 \pm 0.022$ & $0.11 \pm 0.055$ & $0.30 \pm 0.093$ \\
WT12W & $0.58 \pm 0.087$ & $0.09 \pm 0.025$ & & $0.33 \pm 0.073$ \\
HL & $0.20 \pm 0.058$ & $0.10 \pm 0.026$ & & $0.70 \pm 0.049$ \\
FI & $0.27 \pm 0.067$ & $0.06 \pm 0.024$ & & $0.67 \pm 0.056$ \\
BMD $^{\mathrm{a}}$ (BMD) & $0.53 \pm 0.085(0.65)$ & $0.00 \pm 0.022(0.00)$ & & $0.47 \pm 0.075(0.35)$ \\
BMC $^{\text {a }}$ (BMC) & $0.68 \pm 0.090(0.85)$ & $0.00 \pm 0.021(0.00)$ & & $0.32 \pm 0.080(0.15)$ \\
BA $^{\text {a }}$ (BA) & $0.24 \pm 0.076(0.26)$ & $0.04 \pm 0.028(0.06)$ & & $0.72 \pm 0.063(0.68)$ \\
FAT $^{\mathrm{a}}$ (FAT) & $0.36 \pm 0.085(0.44)$ & $0.06 \pm 0.029(0.05)$ & & $0.58 \pm 0.070(0.51)$ \\
BAT $^{\text {a }}$ (BAT) & $0.28 \pm 0.072(0.34)$ & $0.03 \pm 0.025(0.02)$ & & $0.69 \pm 0.060(0.64)$ \\
SCF $^{\mathrm{a}}$ (SCF) & $0.38 \pm 0.081(0.40)$ & $0.04 \pm 0.025(0.03)$ & & $0.58 \pm 0.069(0.57)$ \\
GF $^{\mathrm{a}}$ (GF) & $0.36 \pm 0.082(0.38)$ & $0.04 \pm 0.026(0.06)$ & & $0.60 \pm 0.069(0.56)$ \\
SPLEEN $^{\mathrm{a}}$ (SPLEEN) & $0.22 \pm 0.065(0.33)$ & $0.03 \pm 0.025(0.03)$ & & $0.75 \pm 0.055(0.64)$ \\
LIVER $^{\mathrm{a}}$ (LIVER) & $0.38 \pm 0.087(0.55)$ & $0.06 \pm 0.030(0.04)$ & & $0.56 \pm 0.072(0.41)$ \\
HEART $^{\mathrm{a}}$ (HEART) & $0.20 \pm 0.061(0.35)$ & $0.02 \pm 0.023(0.00)$ & & $0.78 \pm 0.052(0.65)$ \\
\hline
\end{tabular}


Litter effects (Tab. II) contribute on average 6\% of the total variance of the 15 characters, but are statistically significant only for the three body weight characters (especially WT3W) and HL. Maternal (dam) effects are significant only for the early body weights (WT3W and WT6W) and are shown only for these two characters where they make sizeable contributions. Except for WT3W, residual environmental effects contribute the bulk of the environmental variance for the characters (mean $=0.54$ ) with a high of 0.78 for HEART ${ }^{\mathrm{a}}$. Residual environmental effects average $60.5 \%$ for the DEXA/dissection characters adjusted for body weight, although this mean decreases to $51.6 \%$ when these characters are not adjusted for body weight. Because of the generally low to moderate proportional contributions of heritabilities and litter variances, residual environmental effects account for the largest proportion of the total variance for 10 of the 15 characters.

\subsection{Phenotypic and genetic correlations}

All genetic correlations between each pair of the 15 characters, calculated for the $10 \mathrm{DEXA} /$ dissection characters adjusted (above diagonal) and not adjusted (below diagonal) for body weight, are presented in Table III. Standard errors are not given because of space considerations, but averaged 0.175 (range 0.020-0.250) for correlations of characters adjusted for body weight and 0.149 (range $0.018-0.257$ ) for correlations of characters not adjusted for body weight. Genetic correlations indicated in bold print reached significance $(P<0.05)$ after sequential Bonferroni evaluation.

Genetic correlations calculated for the weight-adjusted characters range from -0.65 to +0.98 , with a mean of absolute values of 0.31 over all characters and also among only the 10 DEXA/dissection characters (Tab. III). Roughly equal numbers are positive/negative in sign, with negative correlations being especially conspicuous for the adiposity characters. Correlations within groups generally were higher (mean of absolute values $=0.58$ ) than those between groups (mean $=0.26$ ), this difference reaching significance $(P<0.0001)$ in Mantel's permutation test. Noticeably high values are seen among the three body weights and the four fat characters although none of the correlations of FAT ${ }^{\mathrm{a}}$ with the other three fat characters $(0.85,0.82,0.93)$ reaches 1.0. As earlier hypothesized, the genetic correlation of HL is positive in sign with FI $(+0.24)$, and negative in sign with FAT $^{\mathrm{a}}(-0.17)$, but neither value is statistically significant. Corresponding genetic correlations calculated for the $10 \mathrm{DEXA} /$ dissection characters without adjustment for body weight are predominantly positive in sign and are consistently higher in magnitude with 
Table III. Genetic correlations estimated for each pair of the 15 characters (above diagonal) and for 10 of the 15 characters without adjustment for body weight at sacrifice (below diagonal). Correlations in bold print are statistically significant $(P<0.05)$ after sequential Bonferroni adjustment.

\begin{tabular}{lcllllllllllllll}
\hline & WT3W & WT6W & WT12W & HL & FI & BMD & BMC & BA & FAT & BAT & SCF & GF & SPLEEN & LIVER & HEART \\
\hline WT3W & & $\mathbf{0 . 6 1}$ & $\mathbf{0 . 7 5}$ & 0.18 & -0.01 & 0.20 & 0.36 & 0.49 & -0.29 & 0.03 & -0.13 & -0.40 & -0.50 & -0.24 & 0.27 \\
WT6W & & & $\mathbf{0 . 9 8}$ & 0.24 & -0.08 & $\mathbf{0 . 4 9}$ & $\mathbf{0 . 5 5}$ & 0.40 & -0.22 & 0.10 & -0.05 & -0.35 & -0.44 & -0.28 & 0.46 \\
WT12W & & & & 0.36 & 0.04 & $\mathbf{0 . 6 1}$ & $\mathbf{0 . 5 3}$ & 0.05 & -0.26 & 0.02 & -0.18 & -0.32 & -0.07 & -0.16 & 0.47 \\
HL & & & & & 0.24 & 0.19 & 0.12 & -0.19 & -0.17 & -0.11 & 0.06 & -0.08 & 0.48 & 0.15 & 0.26 \\
FI & & & & & & 0.02 & 0.16 & 0.41 & $\mathbf{- 0 . 6 5}$ & -0.35 & -0.45 & $-\mathbf{0 . 6 1}$ & 0.18 & 0.30 & 0.17 \\
BMD & 0.39 & $\mathbf{0 . 6 7}$ & $\mathbf{0 . 7 0}$ & 0.27 & -0.06 & & 0.10 & 0.43 & -0.07 & -0.30 & -0.14 & -0.22 & 0.24 & 0.19 & 0.14 \\
BMC & $\mathbf{0 . 6 1}$ & $\mathbf{0 . 7 7}$ & $\mathbf{0 . 7 8}$ & $\mathbf{0 . 4 6}$ & 0.00 & $\mathbf{0 . 9 6}$ & & $\mathbf{0 . 7 2}$ & -0.36 & -0.39 & -0.30 & -0.37 & 0.14 & 0.12 & 0.32 \\
BA & $\mathbf{0 . 8 6}$ & $\mathbf{0 . 7 2}$ & $\mathbf{0 . 6 6}$ & 0.02 & 0.29 & $\mathbf{0 . 7 3}$ & $\mathbf{0 . 8 8}$ & & $\mathbf{- 0 . 6 2}$ & -0.49 & -0.53 & -0.45 & -0.19 & -0.20 & 0.46 \\
FAT & 0.50 & $\mathbf{0 . 7 0}$ & $\mathbf{0 . 5 8}$ & 0.10 & $\mathbf{- 0 . 6 1}$ & $\mathbf{0 . 4 6}$ & $\mathbf{0 . 4 3}$ & -0.01 & & $\mathbf{0 . 8 5}$ & $\mathbf{0 . 8 2}$ & $\mathbf{0 . 9 3}$ & -0.03 & -0.08 & -0.43 \\
BAT & 0.25 & $\mathbf{0 . 5 1}$ & 0.43 & -0.01 & -0.36 & -0.07 & 0.12 & 0.03 & $\mathbf{0 . 8 4}$ & & $\mathbf{0 . 9 5}$ & $\mathbf{0 . 7 2}$ & -0.15 & 0.20 & -0.15 \\
SCF & 0.30 & $\mathbf{0 . 5 7}$ & 0.44 & 0.19 & -0.45 & 0.27 & -0.07 & -0.07 & $\mathbf{0 . 9 6}$ & $\mathbf{0 . 9 5}$ & & $\mathbf{0 . 7 1}$ & 0.02 & 0.17 & -0.18 \\
hl GF & 0.27 & 0.47 & 0.32 & 0.12 & $\mathbf{- 0 . 6 7}$ & 0.22 & 0.25 & -0.19 & $\mathbf{0 . 9 2}$ & $\mathbf{0 . 8 1}$ & $\mathbf{0 . 7 5}$ & & -0.09 & -0.33 & -0.51 \\
SPLEEN & 0.13 & 0.39 & $\mathbf{0 . 5 2}$ & 0.54 & 0.10 & $\mathbf{0 . 5 6}$ & $\mathbf{0 . 5 4}$ & 0.28 & 0.35 & 0.10 & 0.33 & 0.16 & & 0.42 & -0.05 \\
LIVER & 0.52 & $\mathbf{0 . 7 5}$ & $\mathbf{0 . 8 1}$ & 0.32 & 0.11 & $\mathbf{0 . 6 6}$ & $\mathbf{0 . 5 6}$ & $\mathbf{0 . 5 6}$ & 0.53 & 0.40 & 0.48 & 0.27 & $\mathbf{0 . 8 5}$ & & 0.18 \\
HEART & $\mathbf{0 . 8 2}$ & $\mathbf{0 . 7 7}$ & $\mathbf{0 . 7 7}$ & 0.44 & 0.28 & $\mathbf{0 . 4 9}$ & $\mathbf{0 . 6 5}$ & $\mathbf{0 . 7 8}$ & 0.25 & 0.17 & 0.23 & 0.04 & $\mathbf{0 . 4 0}$ & $\mathbf{0 . 6 3}$ & \\
\hline
\end{tabular}


a mean of absolute values of 0.45 . Many more of these correlations involving the adjusted characters reach statistical significance, including some with the three organ weight characters.

Phenotypic correlations between each pair of the 15 characters, calculated for the $10 \mathrm{DEXA} /$ dissection characters adjusted (above diagonal) and not adjusted (below diagonal) for body weight, are presented in Table IV. Standard errors averaged 0.023 (range $0.015-0.024$ ) for correlations of characters adjusted for body weight and 0.022 (range $0.015-0.024$ ) for correlations of characters not adjusted for body weight. Estimates for phenotypic correlations shown in bold print reached significance $(P<0.05)$ after sequential Bonferroni adjustment. Phenotypic correlations among the weight-adjusted characters range from -0.44 to +0.79 , with a mean of absolute values of 0.19 ( 0.18 for DEXA/dissection characters). As with the genetic correlations, there are roughly equal numbers of positive and negative phenotypic correlations $(59+, 46-)$, with many of the negative correlations involving the measures of fat. Corresponding phenotypic correlations calculated for the 10 DEXA/dissection characters without adjustment for body weight are predominantly positive in sign and are consistently higher in magnitude with a mean of absolute values of 0.27 .

In general, genetic correlations tend to be higher than their respective phenotypic correlations. However, the element-wise Pearson (matrix) correlation of phenotypic with genetic correlations of each of the 95 pairs of characters is 0.87 , which is highly significant $(P<0.0001)$ as judged by Mantel's permutation test. This suggests that the patterns of phenotypic and genetic correlations among the characters are quite similar.

\section{DISCUSSION}

The advanced intercross population of mice used in this study provided a unique genetic background for analysis of genetic variability and covariability of traits related to body weight, body composition and energy balance. The original ML and MH selection lines from which this population was derived exhibited a large divergence in heat loss with the ML mean = $107.5 \mathrm{kcal} / \mathrm{kg}^{0.75} /$ day and the MH mean $=179.1$ [44]. Moody et al. [37] exploited this diversity by crossing these lines (MH X ML and MH X C57BL/6) to produce two different populations, the analysis of which showed nine putative heat loss QTL on seven different chromosomes. Collectively, these QTL accounted for 28\% of the residual variance for heat loss [37], approximately representing the realized heritability estimated by Nielsen et al. [41]. 
Table IV. Phenotypic correlations estimated for each pair of the 15 characters (above diagonal) and for 10 of the 15 characters without adjustment for body weight at sacrifice (below diagonal). Correlations in bold print are statistically significant $(P<0.05)$ after sequential Bonferroni adjustment.

\begin{tabular}{|c|c|c|c|c|c|c|c|c|c|c|c|c|c|c|c|}
\hline & WT3W & WT6W & WT12W & HL & FI & BMD & BMC & BA & FAT & BAT & SCF & GF & SPLEEN & LIVER & HEART \\
\hline WT3W & & 0.59 & 0.53 & 0.11 & 0.04 & 0.13 & 0.23 & 0.20 & -0.08 & 0.04 & 0.01 & -0.15 & -0.16 & -0.08 & 0.10 \\
\hline WT6W & & & 0.79 & 0.11 & -0.04 & 0.31 & 0.41 & 0.29 & -0.10 & 0.00 & -0.05 & -0.14 & -0.16 & -0.20 & 0.15 \\
\hline WT12W & & & & 0.17 & -0.06 & 0.31 & 0.36 & 0.12 & -0.06 & 0.00 & -0.07 & -0.02 & 0.02 & -0.15 & 0.17 \\
\hline HL & & & & & 0.04 & 0.08 & 0.02 & -0.04 & 0.00 & 0.01 & 0.06 & 0.02 & 0.13 & 0.06 & 0.06 \\
\hline FI & & & & & & 0.14 & 0.20 & 0.13 & -0.44 & -0.13 & -0.35 & -0.41 & 0.11 & 0.27 & 0.17 \\
\hline BMD & 0.24 & 0.42 & 0.47 & 0.11 & 0.10 & & 0.59 & -0.20 & -0.05 & -0.08 & -0.19 & -0.14 & 0.12 & 0.09 & 0.04 \\
\hline $\mathrm{BMC}$ & 0.41 & 0.61 & 0.61 & 0.14 & 0.11 & 0.78 & & 0.56 & -0.35 & -0.22 & -0.34 & -0.41 & 0.06 & 0.00 & 0.13 \\
\hline BA & 0.36 & 0.47 & 0.43 & 0.04 & 0.09 & 0.08 & 0.69 & & -0.43 & -0.20 & -0.36 & -0.41 & -0.08 & -0.12 & 0.12 \\
\hline FAT & 0.30 & 0.49 & 0.55 & 0.11 & -0.38 & 0.25 & 0.19 & -0.02 & & 0.34 & 0.73 & 0.77 & -0.11 & -0.19 & -0.16 \\
\hline BAT & 0.16 & 0.23 & 0.23 & 0.05 & -0.13 & 0.05 & 0.02 & -0.06 & 0.42 & & 0.34 & 0.29 & -0.07 & 0.05 & -0.03 \\
\hline $\mathrm{SCF}$ & 0.24 & 0.33 & 0.36 & 0.12 & -0.34 & 0.09 & -0.06 & -0.10 & 0.84 & 0.41 & & 0.62 & -0.08 & -0.08 & -0.13 \\
\hline GF & 0.18 & 0.35 & 0.43 & 0.10 & -0.39 & 0.10 & 0.02 & -0.12 & 0.84 & 0.37 & 0.68 & & -0.08 & -0.19 & -0.15 \\
\hline SPLEEN & 0.11 & 0.25 & 0.36 & 0.19 & 0.08 & 0.29 & 0.30 & 0.13 & 0.17 & 0.04 & 0.10 & 0.12 & & 0.29 & 0.02 \\
\hline LIVER & 0.26 & 0.55 & 0.64 & 0.16 & 0.14 & 0.29 & 0.44 & 0.27 & 0.37 & 0.23 & 0.25 & 0.25 & 0.60 & & 0.07 \\
\hline HEART & 0.31 & 0.35 & 0.37 & 0.10 & 0.13 & 0.20 & 0.33 & 0.27 & 0.12 & 0.07 & 0.05 & 0.06 & 0.18 & 0.33 & \\
\hline
\end{tabular}


The heritability of heat loss calculated in the AIL-F11 population was somewhat lower (0.20), but does not differ significantly from 0.28. The AIL-F11 population therefore appears to have reconstituted the large level of genetic variability for heat loss (and presumably other traits) comparable to that originally generated from the divergence of the ML and MH selection lines.

\subsection{Genetic and environmental variances}

Environmental sources of variation in the models for genetic parameter estimation included maternal effects, but these were significant only for body weight in mice three (WT3W) and six weeks of age (WT6W). This is not surprising since many previous studies have shown that maternal effects are highest for body weight in young mice or rats and tend to decline with age $[2,9,31,32]$ although they can persist through adulthood [16]. Because all 13 other characters in this study were measured in mice 12 or more weeks of age, maternal effects presumably had declined to the point where they no longer reached significance. Litter effects were significant for the three body weight characters as well as for HL, although their contribution to the total variance was low for all characters except WT3W. Most of the environmental variance in characters measured in these adult mice therefore was residual in nature, and was not associated with differences among the dams or individual litters.

Heritabilities were generally well estimated, with standard errors less than 0.10 for all characters except body weight at three (WT3W) and six weeks of age (WT6W). These two characters showed heritabilities of 0.53 and 0.58 , values higher than comparable estimates for young mice found in some previous studies with outbred populations $[9,22,31,32]$. On the other hand, Cheverud et al. [11] discovered that QTL identified from an intercross of the Large and Small inbred strains accounted for 56\% and 62\%, respectively, of the variation in body weight in mice three weeks and six weeks of age. The number of QTL controlling body weight increased with age, and rather separate QTL were found to control early (3 to 6 week) versus late (6 to 10 week) growth $[11,48]$. Presumably there are many QTL contributing to the genetic variance in body weight in the AIL-F11 population we used as well, and in fact four such QTL were identified by Moody et al. [37] in their analysis of body weight in a mouse population created from a cross of the MH line with C57BL/6.

Apart from body weights, the highest heritabilites were seen for $\mathrm{BMD}^{\mathrm{a}}$ $(0.53)$ and $\mathrm{BMC}^{\mathrm{a}}(0.68)$. Heritabilities for these characters calculated without adjustment for body weight were even higher $(0.65,0.85)$, suggesting that both BMD and BMC have a genetic association with body weight. These high 
heritabilities are consistent with comparable estimates made from inbred strain comparisons and QTL studies in mice; for example, Beamer et al. [3] calculated a broad-sense heritability of 0.57 for BMD in mice generated from a C57BL/6J X Cast/EiJ intercross. Broad-sense heritability estimates for BMD in humans typically are in the 0.6 to 0.7 range [1], so clearly BMD is a highly heritable character. The heritability for bone area $\left(\mathrm{BA}^{\mathrm{a}}\right)$ was much lower (0.24), and this is somewhat surprising given its positive, moderate to high phenotypic and genetic correlations with $\mathrm{BMC}^{\mathrm{a}}$ (although not $\mathrm{BMD}^{\mathrm{a}}$ ). This character is quite different than the other two bone characters, however, representing the total post-cranial area of the skeleton scanned by DEXA as opposed to its estimated mineral density or content.

Most of the other characters had low to moderate heritabilities. Feed intake (FI) showed a heritability of 0.27 , and this is within the range $(0.20$ to 0.30 ) used by Nielsen et al. [42] to calculate genetic correlations for this character with heat loss in the original selection study with the MH and ML lines. The magnitude of the heritability of feed intake conventionally has been expected to be similar to that for heat loss [41]. Heritabilities for the four weight-adjusted adiposity measures ranged from 0.28 to 0.38 ( 0.34 to 0.44 when calculated without adjustment for body weight), and again these values generally are in line with similar estimates made for various fatness characters in mice [13, 21, 22, 29, 44]. Heritabilities for the three weight-adjusted organ weights tended to be rather low, although all three estimates increased considerably when these characters were not adjusted for body weight. This was particularly true for LIVER which showed the highest heritability $(0.55)$ among those for the three organ weights, presumably reflecting the fact that the genetic correlation of $\mathrm{W} 12 \mathrm{~W}$ is higher with LIVER than with either SPLEEN or HEART (Tab. III). Kramer et al. [29] also discovered a higher heritability for liver weight $(0.45)$ compared with heart or spleen weight $(0.20,0.26)$ in their mouse population derived from an intercross of the Large and Small inbred strains. Jones et al. [27] estimated the heritability for liver mass as 0.53, and for liver mass: body mass as 0.52 , in the original base population used to derive the MH and ML lines.

The heritability estimates made for the 10 DEXA/dissection characters without adjustment for body weight all were higher than those made with adjustment for body weight, and this seems to be associated with the magnitude of the genetic correlation of each character with 12-week body weight. Thus the Pearson correlation of the difference between heritabilities for the 10 characters estimated with and without adjustment with the genetic correlation of each of these characters with body weight is $0.61(P=0.06)$. The only 
exception to this trend appears to be for bone area which shows little difference in heritability estimates whether adjusted/unadjusted for body weight in spite of the moderately high genetic correlation of BA with W12W (+0.66). Elimination of this one character in fact increases the correlation describing this association among the remaining nine characters to $0.78(P=0.013)$.

\subsection{Genetic correlation patterns}

The genetic correlations among the 15 characters varied considerably, although their overall absolute mean was relatively low, especially for the characters calculated with adjustment for body weight (0.31). As evidenced by the significant result in Mantel's test, these correlations did tend to be higher among characters within groups than between groups, and this was especially noticeable for the body weight and adiposity groups. Genetic correlations among body weight measured in mice at different ages have generally been quite high, especially between estimates for the closest age groups [29, 33]. Genetic correlations of body weight in 6- and 12-week-old mice were moderately high with both $\mathrm{BMD}^{\mathrm{a}}$ and $\mathrm{BMC}^{\mathrm{a}}$, and at first glance this seems rather surprising since these bone characters were adjusted for body weight. Adjustment occurred for weight at sacrifice, however, not for earlier weights, and perhaps again this result may reflect the relatively independent genetic control of body weight at early versus later ages $[11,44]$. BMD and BMC themselves were highly genetically correlated when not adjusted for body weight (0.96), so it might be expected that they would show similar trends with body weight.

The genetic correlations among the four adiposity characters were relatively high (ranging from 0.71 to 0.93 ) and are comparable to estimates previously made among different adiposity characters in mice [22]. But none of the three genetic correlations of $\mathrm{FAT}^{\mathrm{a}}$ with $\mathrm{BAT}^{\mathrm{a}}, \mathrm{SCF}^{\mathrm{a}}$ or $\mathrm{GF}^{\mathrm{a}}$ approached unity, suggesting that the three fat pad measures are not entirely adequate substitute measures of overall fatness. The genetic correlation of 0.82 of $\mathrm{FAT}^{\mathrm{a}}$ with $\mathrm{SCF}^{\mathrm{a}}$, for example, implies that FAT ${ }^{a}$ shares only about $2 / 3\left(0.82^{2}=0.67\right)$ of its genetic variance with SCFa . Pomp [44] reviewed several QTL studies that used different measures of adiposity, and showed that the QTL discovered often depended on the particular measure used. This sort of result may be due to factors such as statistical detection failure, sexual dimorphism in fat characters and/or measurement at different ages [44]. Clearly we need to know more about the interrelationships among these characters since they evidently are not all the same and assess different aspects of adiposity. 
Three of the four weight-adjusted adiposity measures exhibited a negative genetic correlation with heat loss, and that of HL with FAT ${ }^{\mathrm{a}}(-0.17)$ is close to the -0.14 to -0.08 range estimated by Nielsen et al. [42] for this association. We had predicted that adiposity and heat loss should be negatively genetically correlated because mice in the $\mathrm{MH}$ and ML selection lines from which the AIL-F11 population was derived exhibited opposite patterns of heat loss and body fat percentage $[36,42]$. Although all four genetic correlations are quite low in magnitude, Moody et al. [37] found some preliminary evidence of possible common QTL for both FAT ${ }^{\mathrm{a}}$ and $\mathrm{BAT}^{\mathrm{a}}$ with HL in mice derived from a cross of the $\mathrm{MH}$ line with the BL inbred strain, indicating that these characters are not entirely independent.

The genetic correlations of weight-adjusted adiposity with 3-, 6- and 12week body weights were generally negative, but were all positive in sign when adiposity was not adjusted for body weight. While this trend was expected given the known association of body weight with adiposity [44], it was somewhat surprising that the genetic correlations of unadjusted adiposity with body weights were only moderately high in magnitude. The highest genetic correlation of SCF with body weight (WT6W), for example, was 0.57, a value considerably less than that of 0.92 found by Kramer et al. [29] for weight of the reproductive fat pad (equivalent to GF) and body weight at sacrifice. However, we used body weight at sacrifice only as a covariate and not as a dependent variable in our analysis, and presumably the genetic correlations of these adiposity measures would have been higher with body weights at these later ages rather than at the earlier ages. It is also possible that the genetic association of adiposity with body weight in mice from the AIL-F11 population may be less than that for the Large X Small intercross population used by Kramer et al. [29], especially since it originated from the MH and ML selection lines of mice which did not show a significant divergence in body weight [42].

FAT $^{\mathrm{a}}$ exhibited a noticeably high negative genetic correlation with FI, and at first glance this seems counterintuitive since we would typically expect a positive genetic association between raw feed intake and overall fat [22]. We assessed feed intake by adjusting raw FI by metabolic body weight, however, and this measure of FI exhibited a correlated response opposite to that shown by fat percentage in the ML and ML selection lines [42] from which the AIL-F11 population was derived. A negative association of FAT ${ }^{\mathrm{a}}$ and FI therefore might be expected in this population, and if real, it would be particularly interesting to know if there are QTL that affect FAT ${ }^{\mathrm{a}}$ in one direction and FI in another (antagonistic pleiotropy), or whether there are combinations of QTL with negative and positive effects on these two characters. With regard to FI itself, 
we predicted that it would be positively genetically correlated with HL. In fact we found a positive genetic correlation of +0.24 for these two characters, a value that is only slightly less than the +0.27 to +0.40 estimates made by Nielsen et al. [42] from the MH and ML selection lines.

Genetic correlations among weight-adjusted characters within the body weight, adiposity, and bone groups were much more prominent than those within the metabolic (HL with FI) and especially the organ weight group where none of the three values reached statistical significance. In fact SPLEEN ${ }^{\mathrm{a}}$, HEART $^{\mathrm{a}}$, and LIVER ${ }^{\mathrm{a}}$ showed no significant genetic associations with any characters, suggesting that these organ weights are largely independent from the other character groups. Mice in the MH selection line tended to have larger liver, spleen, and heart weights compared with mice in the ML or control lines, presumably because of their greater energy intake and expenditure [36], so some association might have been expected with HL and/or FI. But even when not adjusted for weight at sacrifice, the three organ weights showed significant genetic linkages only with characters in the bone and body weight groups. Consistent with this, Leamy et al. [35] found a number of QTL in a backcross population of mice that affected (unadjusted) organ weights or limb lengths independently of each other, but also five QTL that pleiotropically affected both sets of characters.

\subsection{Correlation congruence}

Phenotypic correlations between each pair of the 15 characters were calculated primarily to test their similarity with the genetic correlations both in magnitude and in pattern. The magnitude of the genetic correlations (mean of absolute values $=0.31$; mean of squared values $=0.146$ ) generally was greater than that for the phenotypic correlations (mean of absolute values $=0.19$, mean of squared values $=0.066$ ), as typically has been found in these comparisons [7, 28]. Cheverud [7] surveyed 41 pairs of phenotypic and genetic correlation matrices, and found that the mean of the squared genetic correlation was 0.49 , higher than the mean of the squared phenotypic correlations (0.29). Among those studies with larger effective sample sizes, however, this disparity reduced to 0.06 [7], which is similar to the 0.08 difference between the squared correlations among the 15 characters in this study. Koots and Gibson [28] sampled over 1000 sets of correlations and also found that the difference between phenotypic and genetic correlations decreased as the precision of the estimates of genetic correlations (as assessed by their standard errors) increased. 


\section{CONCLUSIONS}

The production of the AIL-F11 population of mice from the original MH and ML selection lines was successful in preserving a significant level of genetic variability for all 15 characters analyzed in this study. Although the heritabilities for several of these characters were fairly low, all estimates were consistent with results from a number of previous studies involving inbred and outbred mouse strains. Genetic covariance patterns also were as expected, with higher correlations among characters within compared to between groups, and with HL being positively associated with FI but negatively associated with the various adiposity characters. There appeared to be considerable independence of adiposity with body weight, suggesting that the AIL-F11 population is an ideal one for a comprehensive QTL study aimed at identifying genes for adiposity that are independent from those affecting body weight. Such a study should be successful in locating QTL for these and other characters such as those analyzed in this study, and it might be expected that a number of these QTL will show pleiotropic effects on two or more of these characters. Furthermore, this AIL has now progressed into its 16th generation. Accumulation of additional recombinations in the population will enable finer-mapping of QTL and potentially assist in cloning of the underlying genes.

\section{ACKNOWLEDGEMENTS}

We thank Sara Olberding, Jeryl Hauptman, Nancy Jerez, Mark Allan, Lori Messer, Jackie Potts, Joao Rocha, Giovani Bertani, Alex Caetano and Stephanie Wesolowski for assistance with mouse husbandry and phenotypic data collection, and an anonymous reviewer for useful revision suggestions. This research is a contribution of the University of Nebraska Agricultural Research Division (Lincoln, NE; Journal Series No. 14367), and was supported in part by funds provided through the Hatch Act. This research was also supported in part by funds provided by the University of North Carolina at Charlotte, and was partially based upon work supported by the National Science Foundation under Grant No. 0091900 (Nebraska EPSCOR infrastructure improvement grant).

\section{REFERENCES}

[1] Arden N.K., Baker J., Hogg C., Spector T.D., The heritability of bone mineral density, ultrasound of the calcaneus and hip axis length: A study of postmenopausal twins, J. Bone Miner. Res. 11 (1966) 530-534. 
[2] Atchley W.R., Rutledge J.J., Genetic components of size and shape. I. Dynamics of components of phenotypic variability and covariability during ontogeny in the laboratory rat, Evolution 34 (1980) 1161-1173.

[3] Beamer W.G., Shultz K.K., Churchill G.A., Frankel W.N., Baylink D.J., Rosen C.J., Donahue L.R., Quantitative trait loci for bone density in C57BL/6J and CAST/EiJ inbred mice, Mamm. Genome 10 (1999) 1043-1049.

[4] Boldman K.G., Kriese L.A., Van Vleck C.P., Van Tassell C.P., Kachman S.D., A manual for use of MTDFREML. A set of programs to obtain estimates of variances and covariances [Draft], USDA-ARS, Lincoln, NE, 1995.

[5] Brockman G.A., Haley C.S., Renne U., Knott S.A., Schwerin M., Quantitative trait loci affecting body weight and fatness from a mouse line selected for extreme high growth, Genetics 150 (1998) 369-381.

[6] Brockmann G.A., Kratzsch J., Haley C.S., Renne U., Schwerin M., Karle S., Single QTL effects, epistasis, and pleiotropy account for two-thirds of the phenotypic F2 variance of growth and obesity in DU6i x DBA/2 mice, Genome Res. 10 (2000) 1941-1957.

[7] Cheverud J.M., A comparison of genetic and phenotypic correlations, Evolution 42 (1988) 958-968.

[8] Cheverud J.M., The genetic architecture of pleiotropic relations and differential epistasis, in: Wagner G.P. (Ed.), The Character Concept in Evolutionary Biology, Academic Press, San Diego, 2000, pp. 413-436.

[9] Cheverud J.M., Rutledge J.J., Atchley W.R., Quantitative genetics of development: Genetic correlations among age-specific trait values and the evolution of ontogeny, Evolution 37 (1983) 895-905.

[10] Cheverud J.M., Leamy L., Quantitative genetics and the evolution of ontogeny. III. Ontogenetic changes in correlation structure among live-body traits in randombred mice, Genet. Res. 46 (1985) 325-335.

[11] Cheverud J.M., Routman E.J., Duarte F.A.M., van Swinderen B., Cothran K., Perel C., Quantitative trait loci for murine growth, Genetics 142 (1996) 1305-1319.

[12] Cheverud J.M., Routman E.J., Irschick D.K., Pleiotropic effects of individual gene loci on mandibular morphology, Evolution 51 (1997) 2006-2016.

[13] Cheverud J.M., Vaughn T.T., Pletscher L.S., Peripato A.C., Adams E.S., Erikson C.F., King-Ellison K.J., Genetic architecture of adiposity in the cross of LG/J and SM/J inbred mice, Mamm. Genome 12 (2001) 3-12.

[14] Corva P.M., Medrano J.F., Quantitative trait loci (QTLs) mapping for growth traits in the mouse: A review, Genet. Sel. Evol. 22 (2001) 105-132.

[15] Corva P.M., Horvat S., Medrano J.F., Quantitative trait loci affecting growth in high growth (hg) mice, Mamm. Genome 12 (2001) 284-290.

[16] Cowley D.E., Pomp D., Atchley W.R., Eisen E.J., Hawkins-Brown D., Impact of maternal uterine genotype on postnatal growth and adult body size in mice, Genetics 122 (1989) 193-203.

[17] Darvasi A., Soller M., Advanced intercross lines, and experimental population for fine genetic mapping, Genetics 141 (1995) 1199-1207.

[18] Dietz E.J., Permutation tests for association between two distance matrices, Syst. Zool. 32 (1983) 21-26. 
[19] Dodenhoff J., Van Vleck L.D., Kachman S.D., Koch R.M., Parameter estimates for direct, maternal and grandmaternal genetic effects for birth weight and weaning weight in Hereford cattle, J. Anim. Sci. 76 (1998) 2521-2527.

[20] Eisen E.J., Selection for components related to body composition in mice: Direct response, Theor. Appl. Genet. 74 (1987) 793-801.

[21] Eisen E.J., Selection experiments for body composition in mice and rats: A review, Livest. Prod. Sci. 23 (1989) 17-32.

[22] Eisen E.J., Prasetyo H., Estimates of genetic parameters and predicted selection responses for growth, fat and lean traits in mice, J. Anim. Sci. 66 (1988) $1153-1165$.

[23] Falconer D.S., Mackay T.F.C., Introduction to Quantitative Genetics, Longman, Essex, 1996.

[24] Graser H.-U., Smith S.P., Tier B., A derivative free approach for estimating variance components in animal models by restricted maximum likelihood, J. Anim. Sci. 64 (1987) 1362-1370.

[25] Holm S., Olm S., A simple sequentially rejective multiple test procedure, Scand. J. Stat. 6 (1979) 65-70.

[26] Horvat S., Bunger L., Falconer V.M., Mackay P., Law A., Bulfield G., Keightley P.D., Mapping of obesity QTLs in a cross between mouse lines divergently selected on fat content, Mamm. Genome 11 (2000) 2-7.

[27] Jones L.D., Nielsen M.K., Britton R.A., Genetic variation in liver mass, body mass, and liver:body mass in mice, J. Anim. Sci. 70 (1992) 2999-3006.

[28] Koots K.R., Gibson J.P., Realized sampling variances of estimates of genetic parameters and the difference between genetic and phenotypic correlations, Genetics 143 (1996) 1409-1416.

[29] Kramer M.G., Vaughn T.T., Pletscher L.S., King-Ellison K., Adams E., Erikson C., Cheverud J.M., Genetic variation in body weight gain and composition in the intercross of Large (LG/J) and Small (SM/J) inbred strains of mice, Genet. Mol. Biol. 21 (1998) 211-218.

[30] Lander E.S., Kruglyak L., Genetic dissection of complex traits: Guidelines for interpreting and reporting linkage results, Nat. Gen. 11 (1995) 241-247.

[31] Leamy L., Heritability of osteometric traits in a randombred population of mice, J. Hered. 65 (1974) 109-120.

[32] Leamy L., Genetical and maternal influences on brain and body size in randombred house mice, Evolution 42 (1988) 42-51.

[33] Leamy L., Cheverud J.M., Quantitative genetics and the evolution of ontogeny. II. Genetic and environmental correlations among age-specific characters in randombred house mice, Growth 48 (1984) 339-353.

[34] Leamy L.J., Routman E.R., Cheverud J.M., Quantitative trait loci for early and late developing skull characters in mice: a test of the genetic independence of morphological integration, Am. Nat.153 (1999) 201-214.

[35] Leamy L.J., Pomp D., Eisen E.J., Cheverud J.M., Pleiotropy of quantitative trait loci for organ weights and limb bone lengths in mice, Physiol. Genomics 10 (2002) 21-29. 
[36] Moody D.E., Pomp D., Nielsen M.K., Variability in metabolic rate, feed intake and fatness among selection and inbred lines of mice, Genet. Res. 70 (1997) $225-235$.

[37] Moody E.E., Pomp D., Nielsen M.K., Van Vleck L.D., Identification of quantitative trait loci influencing traits related to energy balance in selection and inbred lines of mice, Genetics 152 (1999) 699-711.

[38] Mousel M.R., Stroup W.W., Nielsen M.K., Locomotor activity, core body temperature, and circadian rhythms in mice selected for high or low heat loss, J. Anim. Sci. 79 (2001) 861-868.

[39] Nadeau J.H., Burrage L.C., Restivo J., Pao Y.-H., Churchill G., Hoit B.D., Pleiotropy, homeostasis, and functional networks based on assays of cardiovascular traits in genetically randomized populations, Genome Res. 13 (2003) 2082-2091.

[40] Nagy T.R., Clair A.-L., Precision and accuracy of dual-energy X-ray absorptiometry for determining in vivo body composition of mice, Obes. Res. 8 (2000) 392-398.

[41] Nielsen M.K., Jones L.D., Freking B.A., DeShazer J.A., Divergent selection for heat loss in mice: I. Selection applied and direct response through fifteen generations, J. Anim. Sci. 75 (1997) 1461-1468.

[42] Nielsen M.K., Freking B.A., Jones L.D., Nelson S.M., Vorderstrasse T.L., Huseey B.A., Divergent selection for heat loss in mice: II. Correlated responses in feed intake, body mass, body composition, and number born through fifteen generations, J. Anim. Sci. 75 (1997) 1469-1476.

[43] Peacock M., Turner C.H., Econs M.J., Foroud T., Genetics of osteoporosis, Endocr. Rev. 23 (2002) 303-326.

[44] Pomp D., Genetic dissection of obesity in polygenic animal models, Behav. Genet. 27 (1997) 285-306.

[45] Pomp D., Nielsen M.K., Quantitative genetics of energy balance - lessons from animal models, Obes. Res. 7 (1999) 106-110.

[46] Reid I.R., Relationships among body mass, its components, and bone, Bone 31 (2002) 547-555.

[47] Rice W.R., Analyzing tables of statistical tests, Evolution 43 (1989) 223-225.

[48] Rocha J.E., Eisen E.J., Van Vleck L.D., Pomp D., A large sample QTL study in mice. I: Growth, Mamm. Genome 15 (2004) 83-99.

[49] Rocha J.E., Eisen E.J., Van Vleck L.D., Pomp D., A large sample QTL study in mice. II: Body Composition, Mamm. Genome 15 (2004) 100-113.

[50] Webber J., Energy balance in obesity, P. Nutr. Soc. 62 (2003) 539-543. 